\title{
Hyperglycemic adverse events following antipsychotic drug administration in spontaneous adverse event reports
}

\author{
Yamato Kato ${ }^{1}$, Ryogo Umetsu', Junko Abe ${ }^{1,2}$, Natsumi Ueda', Yoko Nakayama', Yasutomi Kinosada ${ }^{3}$ \\ and Mitsuhiro Nakamura ${ }^{1 *}$
}

\begin{abstract}
Background: Antipsychotics are potent dopamine antagonists used to treat schizophrenia and bipolar disorder. The aim of this study was to evaluate the relationship between antipsychotic drugs and adverse hyperglycemic events using the FDA Adverse Event Reporting System (FAERS) database. In particular, we focused on adverse hyperglycemic events associated with atypical antipsychotic use, which are major concerns.
\end{abstract}

Findings: We analyzed reports of adverse hyperglycemic events associated with 26 antipsychotic drugs in the FAERS database from January 2004 to March 2013. The Standardized Medical Dictionary for Regulatory Activities Queries (SMQ) preferred terms (PTs) was used to identify adverse hyperglycemic events. The number of adverse hyperglycemic reports for the top eight antipsychotic drugs, quetiapine, olanzapine, risperidone, aripiprazole, haloperidol, clozapine, prochlorperazine, and chlorpromazine was 12,471 (28.9\%), 8,423 (37.9\%), 5,968 (27.0\%), 4,045 (23.7\%), 3,445 (31.5\%), 2,614 (14.3\%), 1,800 (19.8\%), and 1,003 (35.7\%), respectively. The reporting ratio increased with co-administration of multiple antipsychotic drugs. For example, adverse hyperglycemic events represented $21.6 \%$ of reports for quetiapine monotherapy, 39.9\% for two-drug polypharmacy, and 66.3\% for three-drug polypharmacy.

Conclusion: Antipsychotic drug polypharmacy may influence signal strength, and may be associated with hyperglycemia. After considering the causality restraints of the current analysis, further robust epidemiological studies are recommended.

Keywords: Antipsychotic drugs, Hyperglycemic adverse events, Adverse event reporting system, Antipsychotic polypharmacy, Antipsychotic monotherapy

\section{Findings}

\section{Background}

Antipsychotics are potent dopamine antagonists used to treat schizophrenia and bipolar disorder [1]. Antipsychotics are categorized as first-generation antipsychotics (typical) and second-generation antipsychotics (atypical). Several studies have reported abnormal glucose metabolism during antipsychotic drug treatment [2-4]. In 2002, diabetic ketoacidosis and coma were reported after olanzapine and quetiapine treatment in Japan [5]. Furthermore, the Food and Drug Administration (FDA) asked manufacturers of

\footnotetext{
* Correspondence: mnakamura@gifu-pu.ac.jp

'Laboratory of Drug Informatics, Gifu Pharmaceutical University, 1-25-4, Daigaku-Nishi, Gifu 501-1196, Japan

Full list of author information is available at the end of the article
}

atypical antipsychotic (AAP) drugs to add a warning to drug labels regarding the increased risk of hyperglycemia and diabetes in 2004 [6]. Thus, hyperglycemia due to antipsychotic drug administration is a serious clinical problem.

According to clinical practice guidelines, AAPs should be used as the first and second line of treatment following the first schizophrenic episode [7-10]. However, treatment resistance and poor efficacy continue to be a significant clinical problem $[2,11,12]$. Since antipsychotic polypharmacy is suggested after failure of antipsychotic monotherapy $[7,9,10]$, multiple antipsychotic drugs have been frequently prescribed $[2,11,13]$. A case-control study indicated that the administration of multiple antipsychotics increases the risk of diabetes mellitus when using AAPs [1]. Several studies also demonstrated the effect of antipsychotic polypharmacy 
on the adverse events; however, the effect of antipsychotic polypharmacy on hyperglycemia remains unclear [11-14].

The FDA Adverse Event Reporting System (FAERS) is a spontaneous reporting system for adverse events. It is the largest and best-known database worldwide, and reflects the realities of clinical practice. Therefore, the FAERS database is one of the primary tools used in pharmacovigilance. The aim of this study was to evaluate the relationship between antipsychotic drugs and adverse hyperglycemic events using the FAERS database. To our knowledge, this study is the first to evaluate the effect of antipsychotic polypharmacy on adverse hyperglycemic events using the FAERS database.

\section{Methods}

Data from the FAERS database from January 2004 to March 2013 were obtained from the FDA website. The
FAERS database structure complies with the International Conference on Harmonization (ICH) E2B. We analyzed 26 antipsychotic drugs associated with hyperglycemia (Table 1). Since drug names in the FAERS database are registered arbitrarily, DrugBank, a reliable drug database, was utilized as a dictionary for the batch conversion and compilation of drug names (Table 2). We followed the FDA's recommendation to adopt the most recent case number in order to identify duplicate reports from the same patient and excluded these from analysis.

Adverse events in the FAERS database are coded according to the terminology preferred by the Medical Dictionary for Regulatory Activities (MedDRA). The Standardized MedDRA Queries (SMQ) index is widely accepted and utilized in the analysis of the FAERS database [15]. We utilized the SMQ for hyperglycemia/new onset diabetes mellitus events (SMQ code: 20000041).

Table 1 Characteristics of antipsychotics in the FDA adverse event reporting system database

\begin{tabular}{|c|c|c|c|c|c|}
\hline Drugs & Total & Cases $^{*}$ & Reporting ratio (\%) & RO & \\
\hline Atypical & 96841 & 21151 & 21.8 & 2.5 & $(2.4-2.5)$ \\
\hline Aripiprazole & 17093 & 4045 & 23.7 & 2.6 & $(2.5-2.7)$ \\
\hline Clozapine & 18217 & 2614 & 14.3 & 1.4 & $(1.3-1.5)$ \\
\hline Olanzapine & 22200 & 8423 & 37.9 & 5.3 & $(5.1-5.4)$ \\
\hline Quetiapine & 43169 & 12471 & 28.9 & 3.5 & $(3.4-3.6)$ \\
\hline Perospirone & 83 & 26 & 31.3 & 3.8 & $(2.4-6.1)$ \\
\hline Risperidone & 22121 & 5968 & 27.0 & 3.1 & $(3.0-3.2)$ \\
\hline Zotepine & 134 & 31 & 23.1 & 2.5 & $(1.7-3.8)$ \\
\hline Typical & 19569 & 3948 & 20.2 & 2.1 & $(2.1-2.2)$ \\
\hline Bromperidol & 48 & 11 & 22.9 & 2.5 & $(1.3-4.9)$ \\
\hline Chlorpromazine & 2812 & 1003 & 35.7 & 4.6 & $(4.3-5.0)$ \\
\hline Fluphenazine & 923 & 234 & 25.4 & 2.8 & $(2.4-3.3)$ \\
\hline Haloperidol & 10922 & 3445 & 31.5 & 3.9 & $(3.7-4.0)$ \\
\hline Levomepromazine & 799 & 166 & 20.8 & 2.2 & $(1.8-2.6)$ \\
\hline Moperone & 0 & 0 & - & - & \\
\hline Nemonapride & 4 & 1 & 25.0 & 2.8 & $(0.3-26.8)$ \\
\hline Perphenazine & 911 & 341 & 37.4 & 5.0 & $(4.4-5.7)$ \\
\hline Pimozide & 246 & 65 & 26.4 & 3.0 & $(2.3-4.0)$ \\
\hline Pipamperone & 207 & 26 & 12.6 & 1.2 & $(0.8-1.8)$ \\
\hline Prochlorperazine & 9103 & 1800 & 19.8 & 2.1 & $(2.0-2.2)$ \\
\hline Propericiazine & 190 & 45 & 23.7 & 2.6 & $(1.9-3.6)$ \\
\hline Spiperone & 1 & 0 & - & - & \\
\hline Sulpiride & 1809 & 331 & 18.3 & 1.9 & $(1.7-2.1)$ \\
\hline Sultopride & 97 & 11 & 11.3 & 1.1 & $(0.6-2.0)$ \\
\hline Thioridazine & 574 & 160 & 27.9 & 3.2 & $(2.7-3.9)$ \\
\hline Tiapride & 336 & 81 & 24.1 & 2.7 & $(2.1-3.4)$ \\
\hline Timiperone & 15 & 4 & 26.7 & 3.0 & $(1.0-9.5)$ \\
\hline Trifluoperazine & 619 & 274 & 44.3 & 6.6 & $(5.7-7.8)$ \\
\hline
\end{tabular}

*With adverse events of interest. 
Table 2 Generic names and brand names of antipsychotics in the DrugBank

\begin{tabular}{|c|c|c|}
\hline & Generic name & Brand name \\
\hline \multicolumn{3}{|l|}{ Atypical } \\
\hline & Aripiprazole & Abilify, Aripiprazole \\
\hline & Clozapine & Clozapin, Clozapine, Clozaril, Fazaclo odt, Leponex \\
\hline & Olanzapine & Olansek, Olanzapine, Symbyax, Zydis, Zyprexa, Zyprexa intramuscular, Zyprexa zydis \\
\hline & Quetiapine & Quetiapine, Quetiapine fumarate, Seroquel, Seroquel xr \\
\hline & Risperdone & Risperdal, Risperdal consta, Risperdal m-tab, Risperdone, Risperidona, Risperidone, Risperidonum, Risperin, Rispolept \\
\hline \multicolumn{3}{|l|}{ Typical } \\
\hline & Chlorpromazine & Chlorpromanyl, Chlorpromazine, Largactil, Thorazine \\
\hline & Haloperidole & $\begin{array}{l}\text { Aloperidin, Aloperidol, Aloperidolo, Apo-haloperidol, Haldol, Haldol la, Haldol solutab, Haloperidol, Haloperidol decanoate, } \\
\text { Haloperidol lactate, Halopidol, Halosten, Keselan, Linton, Novo-peridol, Peridol, Serenace }\end{array}$ \\
\hline & Prochloroperazine & $\begin{array}{l}\text { Buccastem, Chlorperazine, Combid, Compazine, Compro, Emetiral, Novamin, Pasotomin, Prochloroperazine, } \\
\text { Prochlorpemazine, Prochlorperazin, Prochlorperazine, Prochlorperazine edisylate, Prochlorperazine maleate, } \\
\text { Prochlorpromazine, Procloperazine, Proclorperazine, Stemetil, Stemzine, Vertigon }\end{array}$ \\
\hline
\end{tabular}

We selected 93 Preferred Terms (PTs), which are summarized in Table 3.

For signal detection, we calculated the reporting odds ratio (ROR), an established pharmacovigilance index, using a disproportionality analysis. The ROR is calculated as $\mathrm{a}^{*} \mathrm{~d} / \mathrm{b}$ *c (Figure 1). The ROR is the ratio of the odds of reporting a specific adverse event versus all other adverse events for a given drug (antipsychotics), compared to the reporting odds for all other drugs present in the database. RORs were expressed as point estimates with $95 \%$ confidence intervals (CI). The detection of a signal was dependent on the signal indices exceeding a predefined threshold. Safety signals were considered significant when the ROR estimates and the lower limits of the 95\% CI were greater than 2 [16]. We analyzed the effects of monotherapy, two-drug polypharmacy, and three-drug polypharmacy. Data analyses were performed using JMP 9.0 (SAS Institute Inc., Cary, NC, USA).

\section{Results}

The FAERS database contains 4,746,890 reports from January 2004 to March 2013. After excluding duplicates according to the FDA's recommendation and extracting the reports with complete age and gender information, 2,257,902 reports were analyzed. Using the SMQ "hyperglycemia/new onset diabetes mellitus" (SMQ20000041), we identified 241,478 adverse hyperglycemic events. The reporting ratios and RORs (95\% CI) for adverse hyperglycemic events are summarized in Table 1 . The reporting ratios of adverse hyperglycemic events in AAPs and typical antipsychotics (TAPs) were $21.8 \%$ (21151/96841) and $20.2 \%$ (3948/19569), respectively. The number of adverse hyperglycemic events among the top eight reported drugs, quetiapine, olanzapine, risperidone, aripiprazole, haloperidol, clozapine, prochlorperazine, and chlorpromazine, was 12,471 (28.9\%), 8,423 (37.9\%), 5,968 (27.0\%), 4,045 (23.7\%),
3,445 (31.5\%), 2,614 (14.3\%), 1,800 (19.8\%), and 1,003 (35.7\%), respectively. Each reporting ratio and ROR was analyzed based on administration (monotherapy, two-drug combination, and three-drug combination; Table 4). The RORs (95\% CI) for monotherapy with quetiapine, olanzapine, risperidone, aripiprazole, haloperidol, clozapine, prochlorperazine, and chlorpromazine were 2.3 (95\% CI: 2.3-2.4), 3.7 (95\% CI: 3.6-3.8), 1.5 (95\% CI: 1.5-1.6), 1.4 (95\% CI: 1.3-1.5), 2.8 (95\% CI: 2.7-3.0), 1.1 (95\% CI: 1.0-1.1), 2.0 (95\% CI: 1.9-2.1), and 1.6 (95\% CI: $1.3-1.8)$, respectively. In contrast, the RORs (95\% CI) for threedrug combination therapy were 16.5 (95\% CI: 15.118.0), 12.0 (95\% CI: 11.0-13.2), 12.0 (95\% CI: 10.9-13.1), 10.3 (95\%: CI 9.1-11.6), 5.9 (95\% CI: 5.3-6.7), 2.3 (95\% CI: 2.0-2.8), 6.0 (95\% CI: 3.6-10.0), and 5.6 (95\% CI: 4.5-6.9), respectively.

\section{Discussion}

Our results suggest that several antipsychotics increase adverse hyperglycemic events, and that antipsychotic polypharmacy may influence these events using the FAERS database.

In a previous cohort study, olanzapine and clozapine were associated with increased risk for type 2 diabetes $[1,2,17]$. Citrome et al. suggested that exposure to multiple AAPs significantly increased the risk of treatmentemergent diabetes mellitus, as compared to TAPs [1]. However, they discussed that their study design does not permit the quantification of differences between AAPs and the risk of emergent diabetes [1]. Another research group reported that AAP administration results in a small increase, as compared to TAP administration [18]. In our study, the reporting ratio of adverse hyperglycemic events in AAPs (21.8\% [21151/96841]) and TAPs (20.2\% [3948/19569]) were similar. Thus, we could not obtain meaningful results regarding the difference between 
Table 3 Preferred terms associated with adverse hyperglycemia in the Standardized MedDRA Queries (SMQ; 20000041)

\begin{tabular}{|c|c|c|c|c|c|c|}
\hline \multirow[t]{2}{*}{ Preferred terms } & \multirow[t]{2}{*}{ Code } & \multirow[t]{2}{*}{ Total } & \multicolumn{2}{|c|}{ Atypical } & \multicolumn{2}{|l|}{ Typical } \\
\hline & & & Cases $^{*}$ & Reporting ratio (\%) & Cases $^{*}$ & Reporting ratio (\%) \\
\hline Total & & 241478 & 21151 & 8.8 & 3948 & 1.6 \\
\hline Abnormal loss of weight & 10000159 & 532 & 28 & 5.3 & 9 & 1.7 \\
\hline Abnormal weight gain & 10000188 & 134 & 33 & 24.6 & 0 & 0 \\
\hline Acidosis & 10000486 & 1956 & 102 & 5.2 & 44 & 2.2 \\
\hline Altered state of consciousness & 10001854 & 3306 & 303 & 9.2 & 111 & 3.4 \\
\hline Anti-GAD antibody positive & 10059728 & 23 & 2 & 8.7 & 0 & 0 \\
\hline Anti-insulin antibody increased & 10053815 & 51 & 0 & 0 & 0 & 0 \\
\hline Anti-insulin antibody positive & 10053814 & 115 & 0 & 0 & 0 & 0 \\
\hline Anti-insulin receptor antibody increased & 10068226 & 0 & 0 & 0 & 0 & 0 \\
\hline Anti-insulin receptor antibody positive & 10068225 & 3 & 0 & 0 & 0 & 0 \\
\hline Anti-islet cell antibody positive & 10049439 & 4 & 1 & 25 & 0 & 0 \\
\hline Blood 1,5-anhydroglucitol decreased & 10065367 & 0 & 0 & 0 & 0 & 0 \\
\hline Blood cholesterol increased & 10005425 & 10887 & 1648 & 15.1 & 63 & 0.6 \\
\hline Blood glucose abnormal & 10005554 & 1547 & 116 & 7.5 & 12 & 0.8 \\
\hline Blood glucose fluctuation & 10049803 & 2267 & 76 & 3.4 & 6 & 0.3 \\
\hline Blood glucose increased & 10005557 & 35838 & 1398 & 3.9 & 241 & 0.7 \\
\hline Blood insulin abnormal & 10005606 & 7 & 0 & 0 & 0 & 0 \\
\hline Blood insulin decreased & 10005613 & 23 & 1 & 4.3 & 1 & 4.3 \\
\hline Blood lactic acid increased & 10005635 & 826 & 47 & 5.7 & 6 & 0.7 \\
\hline Blood osmolarity increased & 10005697 & 112 & 16 & 14.3 & 3 & 2.7 \\
\hline Blood triglycerides increased & 10005839 & 5404 & 1199 & 22.2 & 35 & 0.6 \\
\hline Body mass index decreased & 10005895 & 59 & 14 & 23.7 & 0 & 0 \\
\hline Body mass index increased & 10005897 & 112 & 29 & 25.9 & 0 & 0 \\
\hline Central obesity & 10065941 & 81 & 7 & 8.6 & 1 & 1.2 \\
\hline Coma & 10010071 & 10703 & 1018 & 9.5 & 253 & 2.4 \\
\hline Dehydration & 10012174 & 27804 & 1067 & 3.8 & 1025 & 3.7 \\
\hline Depressed level of consciousness & 10012373 & 10200 & 819 & 8 & 333 & 3.3 \\
\hline Diabetes complicating pregnancy & 10012596 & 3 & 1 & 33.3 & 0 & 0 \\
\hline Diabetes mellitus & 10012601 & 15780 & 5523 & 35 & 98 & 0.6 \\
\hline Diabetes mellitus inadequate control & 10012607 & 3689 & 825 & 22.4 & 25 & 0.7 \\
\hline Diabetes with hyperosmolarity & 10012631 & 27 & 8 & 29.6 & 0 & 0 \\
\hline Diabetic coma & 10012650 & 1045 & 551 & 52.7 & 1 & 0.1 \\
\hline Diabetic hepatopathy & 10071265 & 0 & 0 & 0 & 0 & 0 \\
\hline Diabetic hyperglycaemic coma & 10012668 & 80 & 7 & 8.8 & 1 & 1.3 \\
\hline Diabetic hyperosmolar coma & 10012669 & 170 & 66 & 38.8 & 7 & 4.1 \\
\hline Diabetic ketoacidosis & 10012671 & 2725 & 1090 & 40 & 26 & 1 \\
\hline Diabetic ketoacidotic hyperglycaemic coma & 10012672 & 32 & 6 & 18.8 & 0 & 0 \\
\hline Fructosamine increased & 10017395 & 5 & 0 & 0 & 0 & 0 \\
\hline Gestational diabetes & 10018209 & 594 & 140 & 23.6 & 15 & 2.5 \\
\hline Glucose tolerance decreased & 10018428 & 13 & 0 & 0 & 0 & 0 \\
\hline Glucose tolerance impaired & 10018429 & 1058 & 260 & 24.6 & 6 & 0.6 \\
\hline Glucose tolerance impaired in pregnancy & 10018430 & 3 & 1 & 33.3 & 0 & 0 \\
\hline Glucose tolerance test abnormal & 10018433 & 36 & 3 & 8.3 & 0 & 0 \\
\hline
\end{tabular}


Table 3 Preferred terms associated with adverse hyperglycemia in the Standardized MedDRA Queries (SMQ; 20000041) (Continued)

\begin{tabular}{|c|c|c|c|c|c|c|}
\hline Glucose urine present & 10018478 & 318 & 23 & 7.2 & 15 & 4.7 \\
\hline Glycosuria & 10018473 & 384 & 140 & 36.5 & 5 & 1.3 \\
\hline Glycosuria during pregnancy & 10018475 & 1 & 0 & 0 & 0 & 0 \\
\hline Glycosylated haemoglobin increased & 10018484 & 2569 & 171 & 6.7 & 11 & 0.4 \\
\hline Hunger & 10020466 & 1575 & 142 & 9 & 8 & 0.5 \\
\hline Hypercholesterolaemia & 10020603 & 2210 & 256 & 11.6 & 26 & 1.2 \\
\hline Hyperglycaemia & 10020635 & 7844 & 1382 & 17.6 & 129 & 1.6 \\
\hline Hyperglycaemic hyperosmolar nonketotic syndrome & 10063554 & 184 & 98 & 53.3 & 7 & 3.8 \\
\hline Hyperglycaemic seizure & 10071394 & 5 & 0 & 0 & 0 & 0 \\
\hline Hyperglycaemic unconsciousness & 10071286 & 10 & 0 & 0 & 0 & 0 \\
\hline Hyperlactacidaemia & 10020660 & 333 & 13 & 3.9 & 5 & 1.5 \\
\hline Hyperlipidaemia & 10062060 & 4585 & 747 & 16.3 & 45 & 1 \\
\hline Hyperosmolar state & 10020697 & 113 & 24 & 21.2 & 3 & 2.7 \\
\hline Hyperphagia & 10020710 & 632 & 157 & 24.8 & 4 & 0.6 \\
\hline Hypertriglyceridaemia & 10020869 & 1127 & 154 & 13.7 & 14 & 1.2 \\
\hline Hypoglycaemia & 10020993 & 10839 & 672 & 6.2 & 99 & 0.9 \\
\hline Hypoinsulinaemia & 10070070 & 1 & 0 & 0 & 0 & 0 \\
\hline Impaired fasting glucose & 10056997 & 67 & 22 & 32.8 & 0 & 0 \\
\hline Impaired insulin secretion & 10052341 & 21 & 0 & 0 & 0 & 0 \\
\hline Increased appetite & 10021654 & 2646 & 494 & 18.7 & 21 & 0.8 \\
\hline Increased insulin requirement & 10021664 & 31 & 2 & 6.5 & 0 & 0 \\
\hline Insulin autoimmune syndrome & 10022472 & 23 & 0 & 0 & 0 & 0 \\
\hline Insulin resistance & 10022489 & 297 & 75 & 25.3 & 0 & 0 \\
\hline Insulin resistance syndrome & 10022490 & 18 & 6 & 33.3 & 0 & 0 \\
\hline Insulin resistant diabetes & 10022491 & 27 & 8 & 29.6 & 0 & 0 \\
\hline Insulin tolerance test abnormal & 10022494 & 3 & 0 & 0 & 0 & 0 \\
\hline Insulin-requiring type 2 diabetes mellitus & 10053247 & 122 & 60 & 49.2 & 0 & 0 \\
\hline Ketoacidosis & 10023379 & 640 & 250 & 39.1 & 3 & 0.5 \\
\hline Ketonuria & 10023388 & 188 & 63 & 33.5 & 5 & 2.7 \\
\hline Ketosis & 10023391 & 100 & 13 & 13 & 3 & 3 \\
\hline Lactic acidosis & 10023676 & 4561 & 119 & 2.6 & 61 & 1.3 \\
\hline Latent autoimmune diabetes in adults & 10066389 & 16 & 0 & 0 & 0 & 0 \\
\hline Lipids increased & 10024592 & 368 & 57 & 15.5 & 1 & 0.3 \\
\hline Loss of consciousness & 10024855 & 28249 & 1750 & 6.2 & 355 & 1.3 \\
\hline Metabolic acidosis & 10027417 & 5512 & 253 & 4.6 & 121 & 2.2 \\
\hline Metabolic syndrome & 10052066 & 392 & 197 & 50.3 & 2 & 0.5 \\
\hline Neonatal diabetes mellitus & 10028933 & 3 & 0 & 0 & 1 & 33.3 \\
\hline Obesity & 10029883 & 2787 & 1211 & 43.5 & 23 & 0.8 \\
\hline Overweight & 10033307 & 442 & 114 & 25.8 & 3 & 0.7 \\
\hline Pancreatogenous diabetes & 10033660 & 6 & 2 & 33.3 & 0 & 0 \\
\hline Polydipsia & 10036067 & 1026 & 271 & 26.4 & 16 & 1.6 \\
\hline Polyuria & 10036142 & 1444 & 197 & 13.6 & 27 & 1.9 \\
\hline Slow response to stimuli & 10041045 & 161 & 37 & 23 & 7 & 4.3 \\
\hline Thirst & 10043458 & 2595 & 224 & 8.6 & 40 & 1.5 \\
\hline
\end{tabular}


Table 3 Preferred terms associated with adverse hyperglycemia in the Standardized MedDRA Queries (SMQ; 20000041) (Continued)

\begin{tabular}{lllllll}
\hline Type 1 diabetes mellitus & 10067584 & 1252 & 590 & 47.1 & 7 & 0.6 \\
Type 2 diabetes mellitus & 10067585 & 5272 & 2862 & 54.3 & 16 & 0.3 \\
Underweight & 10048828 & 111 & 8 & 7.2 & 2 & 1.8 \\
Unresponsive to stimuli & 10045555 & 5657 & 442 & 7.8 & 123 & 2.2 \\
Urine ketone body present & 10057597 & 304 & 31 & 10.2 & 13 & 4.3 \\
Weight decreased & 10047895 & 42275 & 1765 & 4.2 & 466 & 1.1 \\
Weight increased & 10047899 & 30417 & 5070 & 16.7 & 867 & 2.9 \\
\hline
\end{tabular}

*With adverse events of interest.

AAP administration and TAP administration using the reporting ratio of hyperglycemic adverse events.

The lower limits of the ROR 95\% CI for olanzapine, quetiapine, and haloperidol monotherapy were greater than 2 (Table 4). Baker et al. reported that olanzapine (AAP), clozapine (AAP), and risperidone (AAP) were associated with hyperglycemic adverse events, whereas aripiprazole (AAP), haloperidol (TAP), and ziprasidone (AAP) had a low association in the FAERS database. We do not have a conclusive explanation for the differences in reporting ratio between the previous report [19] and our findings. One plausible reason could be differences in the terms selected for adverse hyperglycemic events in the MedDRA database. Our study used 93 PTs, whereas Baker et al. used 24. Additionally, different datasets were used for the analyses. Baker et al. performed their analysis using cumulative subsets from 1968 to 2006, whereas our group utilized datasets from 2004 to 2013.

In this study, each reporting ratio and ROR increased with increasing number of drugs administered (Table 4). The ROR of the three-drug polypharmacy had the highest value for every antipsychotic. Therefore, antipsychoticinduced adverse hyperglycemic events may be influenced by the number of drugs administered. However, the lower limit of the clozapine ROR 95\% CI was less than 2. Since the administration of clozapine is not recommended as a first-line treatment [20], physicians may be unlikely to use clozapine in diabetic patients. Therefore, the signal for adverse hyperglycemic events following clozapine might be not detected. Antipsychotic monotherapy and polypharmacy to treat schizophrenia and bipolar disorder has been compared to understand its risk-benefit profile $[11,14]$. In general, polypharmacy using antipsychotics is not recommended [7-9]. Baker et al. evaluated the adverse events signals for each AAP. However, they did not evaluate the effect of antipsychotic polypharmacy on hyperglycemia. Our results suggest that antipsychotic polypharmacy may influence adverse hyperglycemic events. Therefore, clinician should comply with guidelines [7-10] and monitor for adverse polypharmacy-induced hyperglycemic events.

The mechanism by which antipsychotics induce adverse hyperglycemic events remains unclear. AAPs are associated with clinically significant weight gain, and have raised significant concerns regarding possible association with hyperglycemia and type 2 diabetes $[1,11,18,19]$. Obesity or diabetes may be confounders for adverse hyperglycemic events. However, detailed information, including patient

\begin{tabular}{lccc}
\hline & $\begin{array}{c}\text { Adverse event } \\
\text { of interest }\end{array}$ & $\begin{array}{l}\text { All other adverse } \\
\text { event of interest }\end{array}$ & Total \\
\hline Drug of interest & $\mathrm{a}$ & $\mathrm{b}$ & $\mathrm{a}+\mathrm{b}$ \\
$\begin{array}{l}\text { All other drug of } \\
\text { interest }\end{array}$ & $\mathrm{c}$ & $\mathrm{d}$ & $\mathrm{c}+\mathrm{d}$ \\
\hline Total & $\mathrm{a}+\mathrm{c}$ & $\mathrm{b}+\mathrm{d}$ & $\mathrm{a}+\mathrm{b}+\mathrm{c}+\mathrm{d}$ \\
\hline $\mathrm{ROR}=\frac{\mathrm{a} / \mathrm{c}}{\mathrm{b} / \mathrm{d}}=\frac{\mathrm{a} \mathrm{d}}{\mathrm{b} \mathrm{c}}$ & & \\
$95 \% \mathrm{Cl}=\mathrm{e}^{\ln (\mathrm{ROR}) \pm 1.96 \sqrt{\frac{1}{\mathrm{a}}+\frac{1}{\mathrm{~b}}+\frac{1}{\mathrm{c}}+\frac{1}{\mathrm{~d}}}}$ & &
\end{tabular}

Figure 1 Two by two contingency table for analysis. 
Table 4 Reporting ratio and ROR for antipsychotic polypharmacy

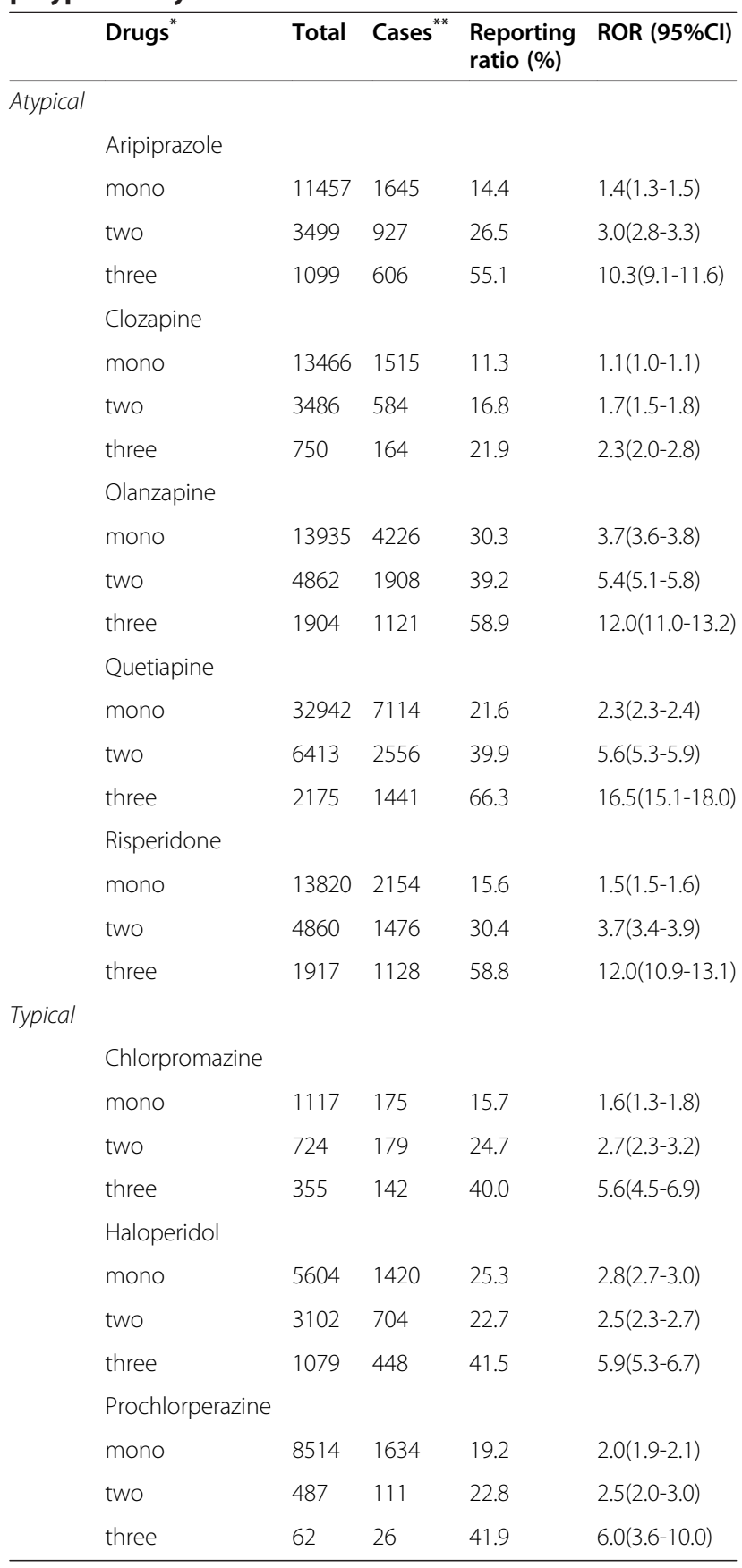

*Monotherapy and polypharmacy of each antipsychotic.

**With adverse events of interest.

background and diagnosis, is not included in the FAERS database. Therefore, it is difficult to define and stratify the patients investigated.

The FAERS database is subject to various biases, including the exclusion of healthy individuals, the lack of denominator, and confounding factors [21]. Because of these deficits within the spontaneous reporting, ROR do not allow for risk quantification. Rather, the RORs offer a rough indication of the signal strength [21]. Therefore, special attention has to be paid to the interpretation of results from the FAERS database. Other epidemiological studies are required to determine the true risk of adverse hyperglycemic events.

Despite the limitations inherent to spontanesous reporting, we obtained reasonable results in the context of the reported literature. The reporting ratio and ROR suggested an association between antipsychotic drugs and hyperglycemic adverse events, and the reporting ratio was increased with an increase in the number of co-administered antipsychotic drugs. Our study indicates the importance of comparing drug safety profiles using post-marketing realworld data. This information could be useful to improve schizophrenia and bipolar disorder management.

\section{Abbreviations \\ FDA: The Food and Drug Administration; AAP: Atypical antipsychotic; FAERS: The FDA adverse event reporting system; $\mathrm{ICH}$ : The International Conference on Harmonization; MedDRA: The medical dictionary for regulatory activities; SMQ: The Standardized MedDRA queries; PT: Preferred terms; ROR: Reporting odds ratio; Cl: Confidence intervals; TAP: Typical antipsychotic. \\ Competing interests \\ JA is an employee of Medical Database. The rest of the authors have no competing interests.}

\section{Authors' contributions \\ YK: conceived of the study and conducted the statistical analysis and manuscript writing. RU: helped to interpretation of data and conduct statistical analyses. JA: participated in the design of the study and helped to conduct statistical analyses. NU: helped to conduct statistical analyses. YN: participated in the design of the study. YK: made contributions to conception and design of the study. MN: conceived of the study, and participated in its design and helped to draft the manuscript. All authors read and approved the final manuscript.}

\section{Acknowledgements}

This research was partially supported by JSPS KAKENHI Grant Number 24390126.

\section{Author details}

'Laboratory of Drug Informatics, Gifu Pharmaceutical University, 1-25-4, Daigaku-Nishi, Gifu 501-1196, Japan. ${ }^{2}$ Medical Database Co., LTD, 3-11-10, Higashi, Sibuya Ward, Tokyo 150-0011, Japan. ${ }^{3}$ Department of Biomedical Informatics, Gifu University Graduate School of Medicine, 1-1 Yanagido, Gifu 501-1196, Japan.

Received: 16 December 2014 Accepted: 23 March 2015

Published online: 16 April 2015

\section{References}

1. Citrome L, Jaffe A, Levine J, Allingham B, Robinson J. Relationship between antipsychotic medication treatment and new cases of diabetes among psychiatric inpatients. Psychiatr Serv. 2004;55:1006-13.

2. Lieberman JA, Stroup TS, McEvoy JP, Swartz MS, Rosenheck RA, Perkins DO, et al. Effectiveness of antipsychotic drugs in patients with chronic Schizophrenia. N Engl J Med. 2005;353:1209-23.

3. Haupt DW, Newcomer JW. Hyperglycemia and Antipsychotic medications. J Clin Psychiatry. 2001;62 Suppl 27:15-26.

4. Scheen AJ, De Hert MA. Abnormal glucose metabolism in patients treated with antipsychotics. Diabetes Metab. 2007;33:169-75.

5. Pharmaceuticals and Medical Devices Agency, Japan. Dear Healthcare Professional Letters. [http://www.pmda.go.jp/safety/info-services/drugs/ calling-attention/esc-rsc/0001.html] 
6. U.S. Food and Drug Administration. Warning about hyperglycemia and atypical antipsychotic drugs. FDA Patient Safety News: Show \#28, June 2004. [http://www.fda.gov/downloads/Safety/FDAPatientSafetyNews/ UCM417797.pdf]

7. Lehman AF, Lieberman JA, Dixon LB, McGlashan TH, Miller AL, Perkins DO, et al. Practice guideline for the treatment of patients with schizophrenia, second edition. Am J Psychiatry. 2004;161 Suppl 2:1-56.

8. Stahl SM, Morrissette DA, Citrome L, Saklad SR, Cummings MA, Meyer JM, et al. "Meta-guidelines" for the management of patients with schizophrenia. CNS Spectr. 2013;18:150-62.

9. Buchanan RW, Kreyenbuhl J, Kelly DL, Noel JM, Boggs DL, Fischer BA, et al. The 2009Schizophrenia PORT psychopharmacological treatment recommendations and summary statements. Schizophr Bull. 2010;36:71-93.

10. Stahl SM. Emerging guidelines for the use of antipsychotic polypharmacy. Rev Psiquiatr Salud Ment. 2013;6:97-100.

11. Correll CU, Rummel-Kluge C, Corves C, Kane JM, Leucht S. Antipsychotic Combinations vs Monotherapy in Schizophrenia: A meta-analysis of randomized controlled trials. Schizophr Bull. 2009;35:443-57.

12. Jones PB, Barnes TR, Davies L, Dunn G, Lloyd H, Hayhurst KP, et al. Randomized controlled trial of the effect on Quality of Life of second- vs first-generation antipsychotic drugs in schizophrenia: Cost Utility of the Latest Antipsychotic Drugs in Schizophrenia Study (CUtLASS 1). Arch Gen Psychiatry. 2006;63:1079-87.

13. Faries D, Ascher-Svanum H, Zhu B, Correll C, Kane J. Antipsychotic monotherapy and polypharmacy in the naturalistic treatment of schizophrenia with atypical antipsychotics. BMC Psychiatry. 2005;5:26.

14. Correll CU, Gallego JA. Antipsychotic polypharmacy: a comprehensive evaluation of relevant correlates of a long-standing clinical practice. Psychiatr Clin North Am. 2012;35:661-81.

15. Medical Dictionary for Regulatory Activities. Introductory Guide for Standardised MedDRA Queries (SMQs) Version 17.0. [http://www.meddra org/sites/default/files/guidance/file/smq_intguide_17_0_english.pdf]

16. Bate A, Evans SJ. Quantitative signal detection using spontaneous ADR reporting. Pharmacoepidemiol Drug Saf. 2009;18:427-36.

17. Nielsen J, Skadhede S, Correll CU. Antipsychotics Associated with the development of Type 2 diabetes in antipsychotic-Naive Schizophrenia patients. Neuropsychopharmacology. 2010;35:1997-2004.

18. Smith M, Hopkins D, Peveler RC, Holt RI, Woodward M, Ismail K. First- V. second-generation antipsychotics and risk for diabetes in schizophrenia: systematic review and meta-analysis. Br J Psychiatry. 2008;192:406-11.

19. Baker RA, Pikalov A, Tran QV, Kremenets T, Arani RB, Doraiswamy PM. Atypical antipsychotic drugs and diabetes mellitus in the US Food and Drug Administration Adverse Event database: a systematic Bayesian signal detection analysis. Psychopharmacol Bull. 2009;42:11-31.

20. Morrissette DA, Stahl SM. Treating the violent patient with psychosis or impulsivity utilizing antipsychotic polypharmacy and high-dose monotherapy. CNS Spectr. 2014;19:439-48.

21. Van Puijenbroek EP, Bate A, Leufkens HG, Lindquist M, Orre R, Egberts AC. A comparison of measures of disproportionality for signal detection in spontaneous reporting systems for adverse drug reactions. Pharmacoepidemiol Drug Saf. 2002;11:3-10.

\section{Submit your next manuscript to BioMed Central and take full advantage of:}

- Convenient online submission

- Thorough peer review

- No space constraints or color figure charges

- Immediate publication on acceptance

- Inclusion in PubMed, CAS, Scopus and Google Scholar

- Research which is freely available for redistribution

Submit your manuscript at www.biomedcentral.com/submit 